\title{
Evaluating Different Methods of Toxoplasma Gondii Detection in Peripheral Blood
}

Rubens Belfort ${ }^{1}$, Jordan Isenberg ${ }^{2}$, Bruno F. Fernandes ${ }^{2}$, Sebastian DiCesare ${ }^{2}$, Rubens Belfort $\mathrm{Jr}^{1}$, Miguel N. Burnier Jr ${ }^{3}$

1. Vision Institute, Department of Ophthalmology, Federal University of São Paulo (UNIFESP) 2. The Henry C. Witleson Ocular Pathology Laboratory, McGill University, McGill University Health Center 3. The Henry C. Witleson Ocular Pathology Laboratory, McGill University; Vision Institute, Department of Ophthalmology, Federal University of São Paulo (UNIFESP)

$\square$ Corresponding author: Rubens Belfort, rubens@belfort.med.br

Disclosures can be found in Additional Information at the end of the article

\section{Abstract}

Introduction: The mechanisms involved in the virulence and recurrences of ocular toxoplasmosis are poorly understood. New detection methods are necessary to diagnose atypical cases and to better understand recurrences. A sensitive method to identify Toxoplasma gondii in peripheral blood of patients is necessary.

Purpose: To detect the presence of T. gondii in peripheral human blood using immunohistochemistry and PCR.

Methods: Yellow fluorescent protein RH strain of T. gondii was cultured on fibroblast monolayer for 5 weeks. Cytospins and whole blood smears from spiked blood were stained using monoclonal antibodies on an automated Ventana. For real time PCR, different concentrations of T. gondii were added to human blood and DNA was extracted. PCR was performed targeting a $62 \mathrm{bp}$ fragment of the B1 gene or the 529bp fragment. The primers used were: B1 Forward 5'CTA GTA TCG GTG CGG CAA TGT -3'and Reverse 5'- GGC AGC GTC TCT TCC TCT TTT -3'. For the 529 bp fragment primers used were 5'- CGC TGC AGG GAG GAA GAC GAA AGT TG- 3 ' and reverse 5' - CGC TGC AGA CAC AGT GCA TCT GAA TT- 3'. All assays were run in triplicate.

Results: The qPCR was able to detect fewer parasites than immunohistochemistry. Moreover, qPCR targeting the $529 \mathrm{bp}$ fragment was better than the B1 gene, being able to detect parasites in concentrations as low as 1 toxo/mL.

Conclusions: The qPCR method using the 529bp target proved to be more sensitive than qPCR using the B1 gene target and cytospins or blood smears using immunohistochemistry.

Received 01/04/2013

Review began 01/05/2013

Published 01/18/2013

\section{(๑) Copyright 2013}

Belfort et al. This is an open access article distributed under the terms of the Creative Commons Attribution License CC-BY 3.0., which permits unrestricted use, distribution, and reproduction in any medium, provided the original author and source are credited.
Categories: Genetics, Internal Medicine, Ophthalmology

Keywords: toxoplasma gondii, detection, fluorescence microscopy, trypan blue exclusion test, toxoplasmosis, ocular, pcr, parasite detection, peripheral blood

\section{Introduction}

Toxoplasmosis is an infection caused by the obligate intracellular parasite, Toxoplasma gondii. It can be acquired either congenitally or by ingestion of food or water contaminated with 
oocysts shed by cats or by eating undercooked or raw meat containing tissue cysts [1-2]. T. gondii infects up to a third of the world's population [2-3]. It is responsible for most of the world's infectious uveitis cases [2-3]. In some populations, up to $50 \%$ of all cases of posterior uveitis is attributed to the disease [3-4].

The majority of toxoplasmosis patients are asymptomatic, with the most common clinical manifestations being lymphadenopathy and retinochoroiditis. Ocular toxoplasmosis can be seen in the setting of congenital or postnatally acquired disease as a result of an acute or recurrent infection [5]. The diagnosis of ocular toxoplasmosis is mainly based on clinical features, particularly in immune-competent patients. Atypical clinical presentations may be seen in both immune-competent and immune-compromised patients, complicating the diagnoses of ocular toxoplasmosis [6]. Given the high rate of prevalence and that the ocular involvement usually presents months to years after the acute disease, positive anti-T. gondii serology is not diagnostic in patients with ocular toxoplasmosis. The severity of the ocular lesion and its propensity to reoccur varies among patients. This may be due to the strain of $\mathrm{T}$. gondii, chronic reinfection, or the genetic characteristics of certain patient populations [7].

The pathogenesis, ocular toxoplasmosis, is not well-understood, especially regarding severity of the disease and recurrence of retinal lesions. Ocular recurrences could derive from activation of retinal cysts or from cysts in other organs that become active causing parasitemia. Another theory is that recurrences in humans could be induced by reinfection with a different strain, which has been substantiated in a mouse model [8]. It has therefore been suggested that the recurrence could be a systemic event and that parasites could be identified in peripheral blood.

Nevertheless, the diagnosis of ocular toxoplasmosis relies mainly on ophthalmological examination, but biological approaches are particularly useful in patients with atypical lesions. The use of serological tests for the demonstration of antibodies to T. gondii is the standard in laboratory diagnostics, a panel of which is useful in establishing whether the individual has been infected recently or in the distant past [9]. Molecular biology assays, such as polymerase chain reaction (PCR), are critical for the diagnosis of the congenital infection [10-11]. It has also been playing an increasing more relevant role in the diagnosis of the ocular disease [12-13], in food safety $[7,14]$ and environmental testing $[15-16]$ for the parasite. In order to validate the diagnostic value of this technology, it is important to determine the number of T. gondii in a titration curve of positive controls. Only that will allow the researcher to determine with maximum precision the number of parasites in the sample from a patient. Furthermore, such titration allows for the sensitivity of downstream PCR reaction to be established.

The trypan blue exclusion test is a rapid and simple technique, which determines the number of viable cells present in a cell suspension and is the "gold standard" in identifying T. gondii in a cellular suspension [17]. The trypan blue exclusion test is based on the principle that live cells possess intact cell membranes that exclude the dye, whereas dead cells do not. The observer then notes either a clear or blue-colored cytoplasm indicating cellular viability [18]. Similarly, yellow fluorescent protein (YFP)-expressing T. gondii tachyzoites present endogenous reporters allowing for the visualization of viable parasites in a cell suspension by fluorescence microscopy [19].

A reliable, clinically available method demonstrating T. gondii in the peripheral blood of patients with systemic and ocular toxoplasmosis could help to establish diagnosis in atypical cases, monitor efficacy of treatment and better explain the mechanism of disease and recurrence. We sought to investigate the reliability of both methods in determining the concentration of T. gondii in a cell suspension and demonstrate that the fluorescence microscopy method is an accurate way to train researchers in the counting of the parasite and then compare the sensitivity of three different methods of T. gondii detection in peripheral 
blood; whole blood smears, immunohistochemistry, and PCR.

\section{Materials And Methods}

\section{T. gondii cell culture and quantification}

Yellow fluorescent protein (YFP)-expressing RH strain T. gondii tachyzoites (Boris Striepen, $\mathrm{PhD}$, University of Georgia, Athens, GA, USA) were kept on a monolayer of vero cells maintained in DMEM medium supplemented with $10 \%$ heat-inactivated fetal bovine serum (FBS), $1 \%$ penicillin-streptomycin (Invitrogen, Burlington, ON, Canada), and L-glutamin at $37^{\circ} \mathrm{C}, 5 \% \mathrm{CO}_{2}$. Tachyzoites were harvested when approximately $80 \%$ of the monolayer was lysed. After centrifugation at $2800 \mathrm{RCF}$ for 15 minutes at room temperature, the pellet was resuspended in $2 \mathrm{~mL}$ of supplemented medium. A 1:1 mixture of YFP-expressing tachyzoites and was prepared and left to incubated for 10 seconds. Quantification was performed by counting the number of unstained, therefore viable, crescent-shaped cells in a Neubauer hemacytometer to determine parasite concentration. Serial dilutions were made from the original solution: $10^{5}$, $10^{4}, 10^{3}, 10^{2}, 10^{1}$ and $10^{-1}$ parasites per milliliter. Quantification of the parasites by fluorescence microscopy was carried out on the same sample subsequent to the trypan blue exclusion test. Parasites were counted at magnifications of 200x for both the trypan blue exclusion test and fluorescence microscopy. Fluorescence was confirmed using an inverted fluorescent microscope (Nikon Eclipse TE2000-U). The procedure was performed six times by three different pathologists.

\section{Peripheral blood smear}

Following methanol immersion, patient blood was fixed to a slide and was air dried prior to counting.

\section{Identification of T. gondii in peripheral blood by immunohistochemistry}

Samples of $3 \mathrm{~mL}$ of blood from a healthy volunteer were spiked with $1 \mathrm{~mL}$ solution of different parasite concentrations. After 30 minutes of incubation at $25^{\circ} \mathrm{C}$ the samples were submitted to either whole blood smear or white layer cytospins on a Cytospin3 machine (Shandon Scientific Limited, Astmoor, UK). White blood cells were separated using Ficoll (Ficoll Paque Plus Amersham Bioscience) and $250 \mu \mathrm{L}$ of cell suspension spun at $30 \mathrm{RCF}$ for three minutes onto a slide. Immunohistochemistry was performed using the Ventana BenchMark LT (Ventana Medical System Inc., Arizona, USA). Slides were incubated with rabbit polyclonal T. gondii Ab-1 antibody (Lab Vision Corp., Fremont, CA, USA) at a dilution of $1: 30$ for 30 minutes at $37^{\circ} \mathrm{C}$, followed by the application of biotinylated secondary antibody for eight minutes at $37^{\circ} \mathrm{C}$ and then an avidin/streptavidin enzyme conjugate complex for eight minutes at $37^{\circ} \mathrm{C}$. Finally, the antibody was detected by Fast Red chromogenic substrate and counterstained with haematoxylin.

\section{Detection of T. gondii via quantitative real-time PCR method}

A mixture containing $1 \mathrm{~mL}$ blood from healthy volunteers and $1 \mathrm{~mL}$ of a media suspension containing different concentrations of T. gondii $10^{4} 10^{3}, 10^{2}, 10^{1}$ and $10^{-1}$ was prepared. DNA was extracted using the QIAamp DNA Blood Midi/Maxi Kit (QIAGEN, Mississauga, ON, Canada) as per the manufacturer's recommendations. The concentration of DNA in the samples was determined using a NanoDrop ND-1000 spectrophotometer (NanoDrop Technologies, Wilmington, DE, USA). The $260 / 280$ and $260 / 230 \mathrm{~nm}$ ratios were used to evaluate DNA purity. Quantification of two genomic targets were compared, the B1 gene [20] and the $529 \mathrm{bp}$ sequence [21]. The primers used were: B1: Forward 5'- TCC CCT CTG CTG GCA AAA AGT -3' 
and Reverse 5'- AGC GTT CGT GGT CAA CTA TCG ATT G -3'. 529bp: 5'- CGC TGC AGG GAG GAA GAC GAA AGT TG-3' and Reverse 5'- CGC TGC AGA CAC AGT GCA TCT GGA TT- 3' (Integrated DNA Technologies Inc., San Jose, CA, USA). Targets were quantified by real-time PCR using QuantiTect one-step SYBR Green PCR method (Qiagen, Mississauga, ON, Canada). A Chromo4 Thermocycler (MJ Research, Waltham, MA, USA) was used for all experiments and was programmed for a $95^{\circ} \mathrm{C} 15$ minutes hot start followed by 44 cycles at $94^{\circ} \mathrm{C}$ for 15 seconds, $58^{\circ} \mathrm{C}$ for 30 seconds and $72^{\circ} \mathrm{C}$ for 60 seconds. The elongation temperature was determined experimentally. Each reaction was followed by a melting curve 60 to $90^{\circ} \mathrm{C}, 1^{\circ} \mathrm{C}$ per second. Beta-actin was used as a positive internal control and for normalization. All results were analyzed using the GeneEx software. Five microliters of PCR product were separated on a $1 \%$ agarose gel and visualized by ethidium bromide staining.

\section{Results}

\section{T. gondii quantification}

Counting by the trypan blue exclusion test yielded an average of 2.90 million parasites (ranging from $1.8-4.0$ million) per milliliter in cell suspension whereas, counting by fluorescence microscopy yielded an average of 6.18 million parasites (ranging from $5.90-6.45$ ) per milliliter in the same sample. Fluorescence microscopy revealed more tachyzoites than the trypan blue exclusion method; the exclusion test underestimated the concentration of the cell suspension by 2.13 fold as shown in Figure 1 .
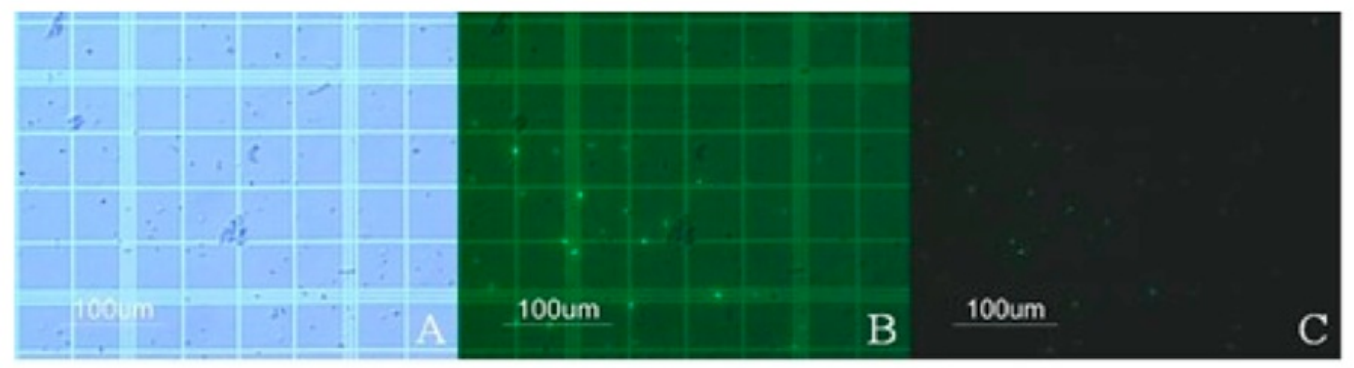

\section{FIGURE 1: Comparison between T. gondii identification by trypan blue exclusion test visualized by light microscopy}

Comparison between T. gondii identification by trypan blue exclusion test visualized by light microscopy (A), a combination of light and fluorescence microscopy illustrating the majority of viable parasites by florescence (B), and the typical visual field as seen when counting the parasite by florescence microscopy (C). All images are of the same cellular suspension, with photos taken in direct succession at a magnification of $200 \mathrm{x}$

Accurate counting of parasites in suspension is challenging for the untrained eye. Tachyzoites are elongated and thin shaped so they might go unnoticed when standing in the upright position inside the counting chamber. Furthermore, the number of parasites might be underestimated if they are clustered together. These dimensional arrangements contribute to unreliable counting. Observers were trained in counting tachyzoites by quantifying first by fluorescence microscopy followed immediately by light microscopy. In this way, a homogenous count was achieved, where one field that yielded an average of 5.9 million parasites (ranging from 5.55 - 6.25 million) per milliliter under fluorescent microscopy resulted in 6.0 million parasites (ranging from 5.3 - 6.7 million) per milliliter under light microscopy. Counting fluorescent parasites contributed to training in identification and determining the concentration of parasites, resulting in a more accurate count. 


\section{Cureus}

\section{Identification of T. gondii in spiked whole blood}

Cytospins were positive for concentrations of $10^{6}, 10^{5}$ and $10^{4}$ toxo/ml while the whole blood smears were positive only for $10^{6}$ and $10^{5}$ toxo/mL. These results were evaluated by four independent pathologists who graded positivity based on stained parasites.

\section{Detection of T. gondii by Quantitative Real-Time PCR Method}

The qPCR method was able to detect parasites in concentrations as low as 0.5 toxo $/ \mathrm{mL}$ of blood. The $529 \mathrm{bp}$ target primers were more sensitive in detecting $T$. gondii when compared with the B1, as shown in Figure 2. Amplification of products resulted in uniform melting curves and fragments with the expected length for each set of primers (Figure 3).

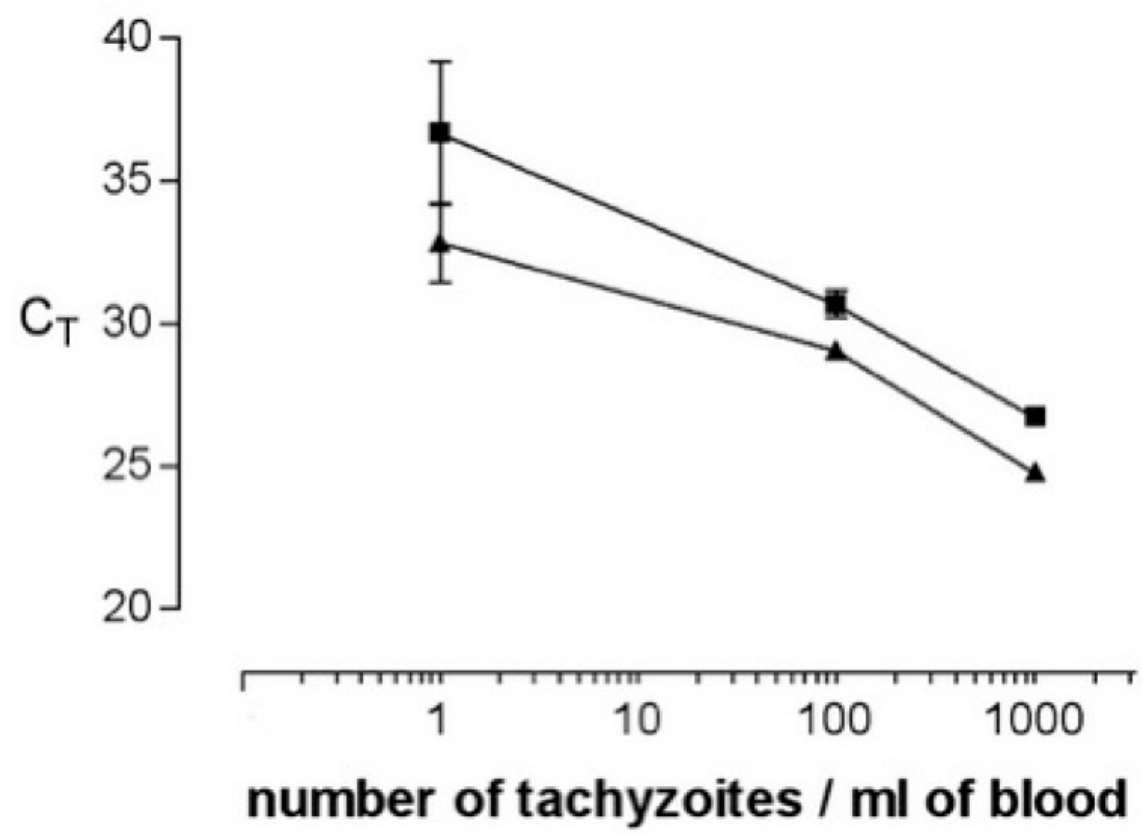

FIGURE 2: Graphic demonstrating the number of cycles during PCR to yield a positive result for T. gondii spiked in whole blood in the concentration shown.

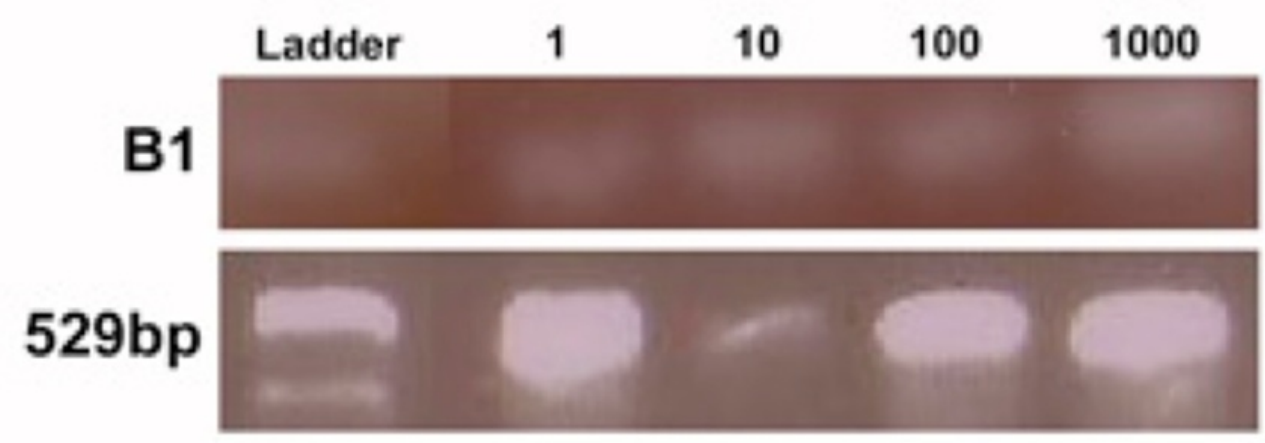




\section{Discussion}

The quantification of T. gondii by fluorescent microscopy is a more accurate method of determining the number of parasites in a cell suspension than the trypan blue exclusion test. The trypan blue exclusion test is a simple and rapid technique that allows for the quantification of cell viability but is not without limitations. The inherent subjectivity of the trypan blue exclusion test coupled with uncertainties that face observers not experienced in counting, such as the low contrast between viable and non-viable cells, variation of exclusion test protocols, and the observer's professional training, all lead to irreproducible results. Counting by fluorescence microscopy allows for not only a precise count but acts also as an aid in training observers to properly employ the trypan blue exclusion test. The underestimation of parasite concentration used to spike blood to determine the sensitivity of a method like PCR could lead to overestimation of the sensibility of such technique. Therefore, we employed YFP transfected parasites in all our experiments, which has resulted in more reliable and reproducible counting.

IHC was able to detect a lower concentration of parasites in cytospins when compared to whole blood smears. T. gondii is able to invade and multiply in all cells and tissue types and has an affinity for muscle and neural cells [22]. Perhaps, the nucleated cells in the peripheral blood attract the parasites in a similar fashion, which could explain the greater sensibility of cytospins when compared to whole blood smears.

In 2000, Held, et al. compared IMC to PCR in the detection of T. gondii in bone marrow recipients and found their sensitivity to be $65 \%$ and $75 \%$, respectively [23]. In contrast, sampling peripheral blood, only high concentrations of parasites could be detected by IHC, demonstrating lower sensitivity when compared to PCR.

qRT-PCR was far more sensitive when compared with the two other methods. Moreover, the 529 bp target being more sensitive than B1 gene because of a greater copy number, confirming previous reports [24]. The sensitivity of PCR to detect T. gondii varies depending with the PCR technique used. In 2006, Calderaro, et al., was able to detect 100 parasites per milliliter using FRET-PCR [25], while Edvinsson, et al., using RT-PCR DNA extracted from spiked blood, was able to detect 1 toxo/ml [24]. Our method could detect as few as 0.5 parasites per milliliter.

To determine the sensitivity of the PCR most published papers add parasite DNA to the reaction, either whole parasite DNA or specific sequences cloned using plasmid [25-28]. This procedure ensures a known concentration per reaction tube. Here, we added tachyzoites to the blood and then proceed with DNA extraction in order to reproduce the exact procedure to which clinical samples will be processed.

\section{Conclusions}

The use of fluorescent microscopy is a valuable tool for identification of T. gondii in a research setting. Fluorescent microscopy is user-friendly and requires no special training, only the identification of fluorescent bodies. By contrast, the trypan blue exclusion test requires an intimate knowledge of the parasite's morphology and its appearance in a cellular suspension. The underestimation of parasites by the trypan blue exclusion test, when used for the ascertainment of PCR sensitivity, for example, leads to a false perception of high sensitivity of 
the molecular test.

After determining the concentration of parasites per unit volume of our cellular suspension, we performed a side-by-side comparison of three methods to detect T. gondii in peripheral blood. All three methods were able to identify T. gondii in peripheral blood. However, the qRT-PCR method proved to be $10^{3}$ times more sensitive than cytospins or blood smears and one particular primer set more sensitive than the other. The B1 sequence is the most widely used amplification target for the diagnosis of toxoplasmosis [27, 29-31], but we have confirmed previous reports that the amplification of the $529 \mathrm{bp}$ target is the most sensitive method for the identification of T. gondii in peripheral blood known to date. We were able to detect as little as 0.5 parasite per milliliter by q-PCR targeting the $529 \mathrm{bp}$ fragment, a level of sensitivity which might allow this technology to be used as a diagnostic tool for toxoplasmosis and, explain the mechanism of disease and recurrence.

\section{Additional Information \\ Disclosures}

Human subjects: All authors have confirmed that this study did not involve human participants or tissue. Animal subjects: All authors have confirmed that this study did not involve animal subjects or tissue. Conflicts of interest: In compliance with the ICMJE uniform disclosure form, all authors declare the following: Payment/services info: This work was funded in part by a grant from Coordenação de Aperfeiçoamento de Pessoal de Nível Superior (Brazil). Financial relationships: All authors have declared that they have no financial relationships at present or within the previous three years with any organizations that might have an interest in the submitted work. Other relationships: All authors have declared that there are no other relationships or activities that could appear to have influenced the submitted work.

\section{Acknowledgements}

Acknowledgements: Prof. Michel Rabinovitch (UNIFESP, Brazil) for the YFP transfected T. gondii.

\section{References}

1. Silveira C, Belfort R Jr, Burnier M Jr, Nussenblatt R: Acquired toxoplasmic infection as the cause of toxoplasmic retinochoroiditis in families. Am J Ophthalmol. 1988, 106:362-4. 10.1001/archophthalmol.2009.354

2. Montoya, J.G., Liesenfeld O: Toxoplasmosis. Lancet. 2004, 363:1965-76. 10.1016/S01406736(04)16412-X

3. Vallochi AL, Muccioli C, Martins MC, et al.: The genotype of Toxoplasma gondii strains causing ocular toxoplasmosis in humans in Brazil. Am J Ophthalmol. 2005, 139:350-1. 10.1016/j.ajo.2004.07.040

4. Soheilian M, Heidari K, Yazdani S, et al.: Patterns of uveitis in a tertiary eye care center in Iran. Ocul Immunol Inflamm. 2004, 12:297-310. 10.1080/092739490500174

5. Silveira C, Belfort R Jr, Muccioli C, et al.: A follow-up study of Toxoplasma gondii infection in southern Brazil. Am J Ophthalmol. 2001, 131:351-4. 10.1016/S0002-9394(00)00830-8

6. Smith, J.R., Cunningham, E.T. Jr : Atypical presentations of ocular toxoplasmosis . Curr Opin Ophthalmol. 2002, 13:387-92.

7. Belfort-Neto R, Nussenblatt V, Rizzo L, et al.: High prevalence of unusual genotypes of Toxoplasma gondii infection in pork meat samples from Erechim, Southern Brazil. An Acad Bras Cienc. 2007, 79:111-4.

8. Dao A, Fortier B, Soete M, et al.: Successful reinfection of chronically infected mice by a different Toxoplasma gondii genotype. Int J Parasitol. 2001, 31:63-5.

9. Montoya, J.G: Laboratory diagnosis of Toxoplasma gondii infection and toxoplasmosis . J 
Infect Dis. 2002, 185:S73-82. 10.1086/338827

10. Hohlfeld P, Daffos F, Costa JM, et al.: Prenatal Diagnosis of Congenital Toxoplasmosis with a Polymerase-Chain-Reaction Test on Amniotic Fluid. N Engl J Med. 1994, 331:695-699. 10.1056/NEJM199409153311102

11. Thalib L, Gras L, Romand S, et al.: Prediction of congenital toxoplasmosis by polymerase chain reaction analysis of amniotic fluid. BJOG. 2005, 112:567-74. 10.1111/j.14710528.2005.00486.x

12. Mahalakshmi B, Therese KL, Madhavan HN, Biswas J: Diagnostic value of specific local antibody production and nucleic acid amplification technique-nested polymerase chain reaction (nPCR) in clinically suspected ocular toxoplasmosis. Ocul Immunol Inflamm. 2006, 14:105-12. 10.1080/09273940500545692

13. Fekkar A, Bodaghi B, Touafek F, et al. : Comparison of immunoblotting, calculation of the Goldmann-Witmer coefficient, and real-time PCR using aqueous humor samples for diagnosis of ocular toxoplasmosis. Journal of Clinical Microbiology. 2008, 46:1965-1967.

10.1128/JCM.01900-07

14. Hill DE, Chirukandoth S, Dubey JP, et al.: Comparison of detection methods for Toxoplasma gondii in naturally and experimentally infected swine. Vet Parasitol. 2006, 14:9-17. 10.1016/j.vetpar.2006.05.008

15. Yang W, Lindquist HD, Cama V, et al.: Detection of Toxoplasma gondii Oocysts in Water Sample Concentrates by Real-time PCR. Appl Environ Microbiol. 2009, 75:3477-83. 10.1128/AEM.00285-09

16. Dumetre, A, M. Darde, M: How to detect Toxoplasma gondii oocysts in environmental samples?. FEMS Microbiology Reviews. 2003, 27:651-661. 10.1128/AEM.00285-09

17. Strober, W: Trypan blue exclusion test of cell viability. Curr Protoc Immunol. 2001, Appendix 3:Appendix 3B. 10.1002/0471142735.ima03bs21

18. Domzig, W., Sequela, J.-P., Binz, H: A Method to Obtain Large Quantities of Toxoplasma gondii Tachyzoites with Extreme Purity. The Journal of Parasitology. 1993, 79:613-615. http://dx.doi.org/10.2307/3283390

19. Gubbels, MJ, Li C, Striepen, B: High-throughput growth assay for Toxoplasma gondii using yellow fluorescent protein. Antimicrob Agents Chemother. 2003, 47:309-16. http://dx.doi.org/10.1128/AAC.47.1.309-316.2003

20. Burg JL, Grover CM, Pouletty P, Boothroyd JC: Direct and sensitive detection of a pathogenic protozoan, Toxoplasma gondii, by polymerase chain reaction. J Clin Microbiol. 1989, 27:178792.

21. Homan WL, Vercammen M, De Braekeleer J, Verschueren H: Identification of a 200- to 300fold repetitive 529 bp DNA fragment in Toxoplasma gondii, and its use for diagnostic and quantitative PCR. Int J Parasitol. 2000, 30:69-75. 10.1016/S0020-7519(99)00170-8

22. Dubey JP, Murrell KD, Fayer R, Schad GA: Distribution of Toxoplasma gondii tissue cysts in commercial cuts of pork. J Am Vet Med Assoc. 1986, 188:1035-7.

23. Held TK, Krüger D, Switala AR, et al. : Diagnosis of toxoplasmosis in bone marrow transplant recipients: comparison of PCR-based results and immunohistochemistry. Bone Marrow Transplant. 2000, 25:1257-62. 10.1038/sj.bmt.1702457

24. Edvinsson B, M. Lappalainen M, Evengard B: Real-time PCR targeting a 529-bp repeat element for diagnosis of toxoplasmosis. Clin Microbiol Infect. 2006, 12:131-6. 10.1111/j.14690691.2005.01332.x

25. Calderaro A, Piccolo G, Gorrini C, et al.: Comparison between two real-time PCR assays and a nested-PCR for the detection of Toxoplasma gondii. Acta Biomed. 2006, 77:75-80.

26. Kasper DC, Sadeghi K, Prusa AR, et al.: Quantitative real-time polymerase chain reaction for the accurate detection of Toxoplasma gondii in amniotic fluid. Diagn Microbiol Infect Dis. 2009, 63:10-15. 10.1016/j.diagmicrobio.2008.09.009

27. Kompalic-Cristo A, Frotta C, Suárez-Mutis M, et al. : Evaluation of a real-time PCR assay based on the repetitive B1 gene for the detection of Toxoplasma gondii in human peripheral blood. Parasitol Res. 2007, 101:619-25. 10.1007/s00436-007-0524-9

28. Cassaing S, Bessières MH, Berry A, et al.: Comparison between two amplification sets for molecular diagnosis of toxoplasmosis by real-time PCR. J Clin Microbiol. 2006, 44:720-4. 10.1128/JCM.44.3.720-724.2006

29. Alfonso Y, Fraga J, Fonseca C, et al.: Molecular diagnosis of Toxoplasma gondii infection in cerebrospinal fluid from AIDS patients. Cerebrospinal Fluid Res. 2009, 6:2. 10.1186/1743- 


\section{Cureus}

8454-6-2

30. Fricker-Hidalgo H, Bulabois CE, Brenier-Pinchart MP, et al.: Diagnosis of toxoplasmosis after allogeneic stem cell transplantation: results of DNA detection and serological techniques. Clin Infect Dis. 2009, 48:e9-15. 10.1086/595709

31. Montoya A, Miró G, Mateo M, et al.: Detection of Toxoplasma gondii in cats by comparing bioassay in mice and polymerase chain reaction (PCR). Vet Parasitol. 2009, 160:159-62. 10.1016/j.vetpar.2008.10.029 